\title{
A Cardinal Function Method of Solution of the Equation $\Delta u=u-u^{3}$
}

\author{
By L. R. Lundin
}

Abstract. The steady-state form of the Klein-Gordon equation is given by

$$
\Delta u=u-u^{3}, \quad u=u(X), \quad X \in R^{3} .
$$

For solutions which are spherically symmetric, $\left(^{*}\right)$ takes the form $\ddot{u}+2 \dot{u} / r=u-u^{3}$, $u=u(r)$, where $r$ is the distance from the origin in $R^{3}$. The function $y=r u$ satisfies

$$
\ddot{y}=y-y^{3} / r^{2} \text {. }
$$

It is known that $\left(^{* *}\right)$ has solutions $\left\{y_{n}\right\}_{n=0}^{\infty}$, where $y_{n}$ has exactly $n$ zeros in $(0, \infty)$, and where $y(0)=y(\infty)=0$.

In this paper, an approximation is obtained for the solution $y_{0}$ by minimizing a certain functional over a class of functions of the form

$$
\sum_{k=-m}^{m} a_{k} \operatorname{sinc}\left[\frac{r-k h_{m}}{h_{m}}\right] \text {. }
$$

It is shown that the norm of the error is $O\left(m^{3 / 8} \exp \left(-\alpha m^{1 / 2}\right)\right)$ as $m \rightarrow \infty$, where $\alpha$ is positive.

1. Introduction. The Klein-Gordon equation

$$
\square \phi=\left(u^{2}-\lambda \phi^{*} \phi\right) \phi, \quad \phi=\phi(X, t), \quad X=\left(x_{1}, x_{2}, x_{3}\right),
$$

is an equation arising in spinor particle theory. Three steady-state forms of (1.1) are

$$
\begin{gathered}
\Delta u=u-u^{3}, \quad u=u(X), \\
\ddot{u}+2 \dot{u} / r=u-u^{3}, \quad u=u(r),
\end{gathered}
$$

and

$$
\ddot{y}=y-y^{3} / r^{2}, \quad y=y(r) .
$$

Equation (1.1) reduces to (1.2) once the following substitutions are made: $\phi=$ $v \exp [i(k \cdot Y-w t)], \beta Y=X, \beta^{2}=u^{2}+k^{2}-w^{2} c^{-2}, u=\beta^{-1} \lambda^{1 / 2} v$. For the radially symmetric case, (1.2) reduces to (1.3), and (1.4) is obtained by setting $y=r u$ in (1.3).

Here, we shall be interested in the solution of the problem

$$
\ddot{y}=y-y^{3} / r^{2}, \quad y(0)=y(\infty)=0,
$$

Received April 26, 1979.

1980 Mathematics Subject Classification. Primary 65L10, 65P05. 
which corresponds to

$$
\ddot{u}+2 \dot{u} / r=u-u^{3}, \quad \dot{u}(0)=u(\infty)=0 .
$$

The problem (1.5) has been considered by many authors [1] , [2], [4], [5] , [10], $[11],[12],[14],[18]$. Nehari [10] proved the existence of solutions to (1.5), and Ryder [13] showed that solutions $y_{n}$ exist, for $0 \leqslant n<\infty$, such that $y_{n}$ has exactly $n$ zeros in $(0, \infty)$. It is these solutions, $y_{0}$ in particular, which this paper concerns.

Problem (1.5) contains obvious numerical difficulties: a nonlinear boundary value problem over an unbounded interval. Initial attempts to use initial value problem techniques are discouraging. Each solution of (1.4) is asymptotic to one of $y=-r$, $y=0$ or $y=r$, as $r \rightarrow \infty$, and apparently (see [2]) no solution of (1.5) is stable. In fact, the evidence indicates that for the collection of initial value problems

$$
\ddot{y}-y-y^{3} / r^{2}, \quad y(0)=0, \quad y^{\prime}(0)=\alpha,
$$

there is a countable collection $\left\{\alpha_{j}\right\}$ with no cluster point in $R$ such that if $\alpha \notin\left\{\alpha_{j}\right\}$, then $|y / r| \rightarrow 1$ as $r \rightarrow \infty$.

Standard proofs of Galerkin methods fail for (1.5), since the derivative of the Lagrangian of (1.4) is neither positive nor negative at a solution to (1.5). However, Chauvette and Stenger [2] do succeed in a difficult proof of their application of the Bubnov-Galerkin method.

Here we consider a variational approach based on the approximation theory developed in [8]. The results of [17] suggests that the rate of convergence, $O\left(\exp \left(-\mathrm{cm}^{1 / 2}\right)\right)$ where $m$ is the number of unknowns, is best possible. Several proofs, which have been deleted in the exposition, are available on request.

2. Important Results. In what follows, we shall make use of the functionals $J(a, b)$ and $G(a, b)$ defined by

$$
J(a, b) y=\int_{a}^{b}\left(\dot{y}^{2}+y^{2}\right) d r
$$

and

$$
G(a, b) y=\int_{a}^{b} y^{4} r^{-2} d r
$$

For simplicity, we write $J$ and $G$ for $J(0, \infty)$ and $G(0, \infty)$, respectively. We also consider the set $S(a, b)$ consisting of all functions $y \in H_{0}^{1}(a, b)$ such that $y\left(c_{y}\right)>0$ for some $c_{y} \in(a, b)$ and

$$
J(a, b) y=G(a, b) y .
$$

THEOREM 2.1 [13]. Let $n \geqslant 0$, and let $S_{n}$ be the collection of all functions $y$ for which the following is satisfied: there exist $\left\{r_{k}\right\}_{k=1}^{n} \subset(0, \infty)\left(\left\{r_{k}\right\}_{k=1}^{n}=\phi\right.$ if $n=0)$ such that $0=r_{0}<r_{1}<\cdots<r_{n}<r_{n+1}=\infty$, and $(-1)^{k} y \in S\left(r_{k}, r_{k+1}\right)$, for $0 \leqslant k \leqslant n$. Then $J$ is minimized over $S_{n}$ by the solution $y_{n}$ of problem (1.5). Further, for each $n \geqslant 0, y_{n} \in C^{1}[0, \infty)$, and $y_{0}$ is the unique minimum of $J$ over $S_{0}$. 
Coffman [1] provides further results on uniqueness and existence, including the following two lemmas.

LEMMA 2.2. For each $\beta>0,(1.4)$ has a unique solution $y=y(r, \beta)$ which satisfies $y \in C^{2}(0, \infty)$ and $\lim _{r \rightarrow 0} r^{-1} y=\beta$. Further, there is at most one $\beta_{0} \in(0, \infty)$ for which $y\left(r, \beta_{0}\right)>0$ on $(0, \infty)$ and $\lim _{r \rightarrow \infty} y\left(r, \beta_{0}\right)=0$.

Lemma 2.3. Let $n \geqslant 0$, and let $b$ be the first zero of $y_{n}$ in $(0, \infty]$. Then $\delta \equiv 0$ is the unique solution of

$$
\delta^{\prime \prime}-\delta+3 r^{-2} y_{n} \delta=0, \quad \delta(0)=\delta(b)=0 .
$$

The theorem which follows is a consequence of Lemma 2.3. Its proof, which employs the Sturm-Liouville theory and Courant's maximum-minimum principle, is deleted.

THEOREM 2.4. Let $b$ and $y_{n}$ be as in Lemma 2.3. Then there exists a constant $\kappa \in(0,2)$ such that for all $\epsilon \in H_{0}^{1}(0, b)$,

$$
6 \int_{0}^{b} y_{n}^{2} \epsilon^{2} r^{-2} d r<(2-\kappa) \int_{0}^{b}\left(\dot{\epsilon}^{2}+\epsilon^{2}\right) d r
$$

3. Whittaker's Cardinal Function. Let $f$ be defined on the real line $R$, and let $h$ be a positive constant. Then the function $C(f, h)$ is defined on the complex plane C by

$$
C(f, h)(z)=\sum_{k=-\infty}^{\infty} f(k h) S(k, h)(z),
$$

where the function $S(k, h)$ is given by

$$
S(k, h)(z)=\left[\pi \frac{z-k h}{h}\right]^{-1} \sin \left[\pi \frac{z-k h}{h}\right] .
$$

The function $C(f, h)$ was introduced by E. T. Whittaker [21], and has been studied further by several authors (see [8], [9], [16], [22]). In this paper, we approximate the solution $y_{0}$ of (1.5) by a function of the form

$$
C_{m}(f, h)=\sum_{k=-m}^{m} f(k h) S(k, h) .
$$

The foundation for such approximations is given in [8]

The results given in [2] may be used to show that for $\alpha=1, y_{0}$ satisfies the conditions on $f$ in the theorem which follows.

THEOREM 3.1 [8]. Let $d, \alpha>0$ and assume $f$ satisfies

(i) $f$ is analytic on $D_{d} \equiv\{x+i y:|y|<d\}$;

(ii) $\int_{-d}^{d}|f(x+i y)| d y \rightarrow 0$ as $x \rightarrow \pm \infty$;

(iii) $\lim _{y \rightarrow d^{-}} \int_{R}|f(x \pm i y)|^{2} d x<\infty$;

(iv) there is a positive constant $C$ such that $|f(x)| \leqslant C e^{-\alpha|x|}$, for all $x \in R$.

Let $\gamma$ be positive and for each positive integer $m$ let $h_{m}=r m^{-1 / 2}$, and let 
$\epsilon_{m}=C\left(f, h_{m}\right)-f$. Then there exist positive constants $K_{1,2}$ and $K_{\infty}$, independent of $m$, such that

$$
\left\|\epsilon_{m}\left(f, h_{m}\right)\right\|_{2}, m^{-3 / 4}\left\|\epsilon_{m}\left(f, h_{m}\right)\right\|_{1,2} \leqslant K_{1,2} \exp \left(-\beta m^{1 / 2}\right),
$$

and

$$
\left\|\epsilon_{m}\left(f, h_{m}\right)\right\|_{\infty}, m\left\|\epsilon_{m}\left(f, h_{m}\right)^{\prime}\right\|_{\infty} \leqslant K_{\infty} m^{1 / 2} \exp \left(-\beta m^{1 / 2}\right),
$$

where $\beta=\min (\pi d / \gamma, \alpha \gamma)$.

It is important to recognize the implications of (3.4) and (3.5). For example, (3.4) says that

$$
\left\|\epsilon_{m}\left(f, h_{m}\right)\right\|_{1,2}=O\left(h_{m}^{-3 / 2} e^{-\gamma / h_{m}}\right) \quad \text { as } h_{m} \rightarrow 0(m \rightarrow \infty),
$$

and so for every positive integer $p$,

$$
\left\|\epsilon_{m}\left(f, h_{m}\right)\right\|_{1,2}=o\left(h_{m}^{p}\right) \quad \text { as } h_{m} \rightarrow 0 .
$$

We complete this section with the presentation of two results which are needed in the computations and in the proof of validity of the approximation scheme. The equalities (3.6)-(3.8) are given in [8], and (3.10) is obtained quite easily from the theory in $[8]$.

THEOREM 3.2. Let $h$ be positive, let $m$ be a positive integer and let

$$
g=\sum_{k=-m}^{m} a_{k} S(k, h)
$$

Then

$$
\int_{R} g^{2}(x) d x=h \sum_{k=-m}^{m} a_{k}^{2}
$$

and

$$
\int_{R}\left[g^{\prime}(x)\right]^{2} d x=h^{-1} \sum_{k=-m}^{m} \sum_{j=-m}^{m} c_{k, j} a_{k} a_{j}
$$

where

$$
c_{k, j}= \begin{cases}\pi^{2} / 3 & \text { if } k=j, \\ 2(-1)^{k+j}(j-k)^{-2} & \text { if } k \neq j\end{cases}
$$

If $a_{0}=0$, then

$$
\int_{R} g^{4}(x) x^{-2} d x=2 h^{-1} \sum_{k=-\infty}^{\infty} k^{-2} g^{4}(k h / 2)
$$

THEOREM 3.3. Let $f, m, h_{m}$ and $\beta$ be as in Theorem 3.1, and assume $f(0)=0$. Then there exists a positive constant $L$, independent of $m$, such that 


$$
\left|\int_{R} f^{4}(x) x^{-2} d x-\int_{R} C_{m}\left(f, h_{m}\right) x^{-2} d x\right| \leqslant L \exp \left(-\beta m^{1 / 2}\right)
$$

Proof. We have

$$
\begin{aligned}
& \left|\int_{R} f^{4}(x) x^{-2} d x-\int_{R} C_{m}\left(f, h_{m}\right) x^{-2} d x\right| \\
& \leqslant\left[\sup _{x \in R \backslash\{0\}} \frac{f^{2}(x)+C_{m}\left(f, h_{m}\right)^{2}(x)}{x^{2}}\right] \int_{R}\left|f^{2}(x)-C_{m}\left(f, h_{m}\right)^{2}\right| d x \\
& \leqslant\left[\sup _{x \in R \backslash\{0\}} \frac{f^{2}(x)+C_{m}\left(f, h_{m}\right)^{2}(x)}{x^{2}}\right]\left\|f+C_{m}\left(f, h_{m}\right)\right\|_{2}\left\|f-C_{m}\left(f, h_{m}\right)\right\|_{2} .
\end{aligned}
$$

Now, $C_{m}\left(f, h_{m}\right)(0)=f(0)=0$, and so the supremum above is finite for each $m$ since each of $f$ and $C_{m}\left(f, h_{m}\right)$ is analytic at $z=0$. Further, (3.4) and (3.5) imply that the supremum and $\left\|f+C_{m}\left(f, h_{m}\right)\right\|_{2}$ are bounded as functions of $m$. So, (3.11) follows from (3.4).

4. Approximating $y_{0}$. The following definitions are suggested since $y_{0}$ is an odd function satisfying Theorems 3.1 and 3.3.

Definition 4.1. For each positive integer $m$, let $h_{m}=\gamma m^{-1 / 2}$, where $\gamma$ is a fixed positive number. Then $W_{m}$ is the collection of all functions $w$ of the form

$$
w(r)=\sum_{k=-m}^{m} a_{k} \operatorname{sinc}\left[\frac{r-k h_{m}}{h_{m}}\right]
$$

which satisfy

$$
a_{-k}=-a_{k} \text { for } 0 \leqslant k \leqslant m,
$$

$$
\begin{aligned}
& J w=G w, \text { where } J, G \text { are as defined in Section 2, } \\
& a_{k} \geqslant 0 \text { for } 1 \leqslant k \leqslant m, \text { and } a_{j} \neq 0 \text { for some } j .
\end{aligned}
$$

Definition 4.2. For each positive integer $m$, let

$$
\begin{gathered}
z_{m}=C_{m}\left(y_{0}, h_{m}\right), \\
\tilde{z}_{m}=\left[J z_{m} / G z_{m}\right]^{1 / 2} z_{m},
\end{gathered}
$$

and let $w_{m}$ be an element of $w_{m}$ for which

$$
J w_{m}=\min _{w \in w_{m}} J w .
$$

The function $w_{m}$ is our $m$ th approximation to $y_{0}$.

We note that $\widetilde{z}_{m} \in W_{m}$, and so $W_{m} \neq \varnothing$. Thus, obtaining $w_{m}$ is equivalent to minimizing a continuous function on a nonempty compact subset of $R^{m}$. Further, with $S_{0}$ defined as in Theorem 2.1, we have $W_{m} \subset S_{0}$, and so $J \tilde{z}_{m}>J y_{0}$.

LEMmA 4.1. There is a positive constant $K$ such that for all $m \geqslant 1$,

$$
0<J w_{m}-J y_{0} \leqslant J \tilde{z}_{m}-J y_{0} \leqslant K m^{3 / 4} \exp \left(-\beta m^{1 / 2}\right),
$$

where $\beta=\min (\pi d / \gamma, \gamma)$ and $d$ is as in Theorem 3.1. 
Proof. We have $w_{m} \in S$, and $w_{m}((m+1) h)=0$. Thus, $w_{m} \not \equiv y_{0}$ and so $0<$ $J w_{m}-J y_{0}$. Since $\tilde{z}_{m} \in w_{m}, J w_{m}-J y_{0} \leqslant J \tilde{z}_{m}-J y_{0}$ follows from (4.7).

To verify the last inequality in (4.8), we first note that

$$
\tilde{z}_{m}-J y_{0}=\left|\left\|\tilde{z}_{m}\right\|_{1,2}^{2}-\left\|y_{0}\right\|_{1,2}^{2}\right| \leqslant\left(\left\|\tilde{z}_{m}\right\|_{1,2}+\left\|y_{0}\right\|_{1,2}\right)\left\|\tilde{z}_{m}-y_{0}\right\|_{1,2} .
$$

Also,

$$
\left\|\tilde{z}_{m}-y_{0}\right\|_{1,2} \leqslant\left\|\tilde{z}_{m}-z_{m}\right\|_{1,2}+\left\|z_{m}-y_{0}\right\|_{1,2} .
$$

We recall from (3.4) that

$$
\left\|z_{m}-y_{0}\right\|_{1,2} \leqslant K_{1} m^{3 / 4} \exp \left(-\beta m^{1 / 2}\right),
$$

for some positive constant $K_{1}$. Further,

$$
\begin{aligned}
\| \tilde{z}_{m}- & z_{m}\left\|_{1,2}=\left|\left[J z_{m} / G z_{m}\right]^{1 / 2}-1\right|\right\|_{m} \|_{1,2} \\
& =\left\|z_{m}\right\|_{1,2}\left|\left(J z_{m}\right)^{1 / 2}-\left(G z_{m}\right)^{1 / 2}\right|\left(G z_{m}\right)^{-1 / 2} \\
& \leqslant\left\|z_{m}\right\|_{1,2}\left(G z_{m}\right)^{-1 / 2}\left\{\left|\left\|z_{m}\right\|_{1,2}-\left\|y_{0}\right\|_{1,2}\right|+\left|\left(G y_{0}\right)^{1 / 2}-\left(G z_{m}\right)^{1 / 2}\right|\right\} \\
& \leqslant\left\|z_{m}\right\|_{1,2}\left(G z_{m}\right)^{-1 / 2}\left\{\left\|z_{m}-y_{0}\right\|_{1,2}+\left|\left(G y_{0}\right)^{1 / 2}-\left(G z_{m}\right)^{1 / 2}\right|\right\} .
\end{aligned}
$$

We know the sequence $\left\{G z_{m}\right\}_{m=1}^{\infty}$ is bounded, since Theorem 3.3 implies $G z_{m} \rightarrow$ $G y_{0}$ as $m \rightarrow \infty$. Furthermore, Theorem 3.3 implies

$$
\left|\left(G z_{m}\right)^{1 / 2}-\left(G y_{0}\right)^{1 / 2}\right| \leqslant K_{2} \exp \left(-\beta m^{1 / 2}\right),
$$

where $K_{2}$ is some positive constant. Thus, there exists a constant $K_{3}$ such that

$$
\left\|\tilde{z}_{m}-z_{m}\right\|_{1,2} \leqslant K_{3} m^{3 / 4} \exp \left(-\beta m^{1 / 2}\right) .
$$

Using (4.11) and (4.13) in (4.10), we arrive at (4.8), since $\left\|\tilde{z}_{m}\right\|_{1,2} \rightarrow\left\|y_{0}\right\|_{1,2}$ in (4.9).

Our next result is now obvious. Its nontrivial proof, which draws on results in functional analysis, is deleted.

THEOREM 4.2. The sequence $\left\{w_{m}\right\}_{m=1}^{\infty}$ converges in $H_{0}^{1}(0, \infty)$ to $y_{0}$. Hence, $\left\|w_{m}-y_{0}\right\|_{1,2},\left\|w_{m}-y_{0}\right\|_{\infty} \rightarrow 0$ as $m \rightarrow \infty$.

Next, we wish to determine the rate of convergence of $w_{m}$ to $y_{0}$. We note that on $S_{0}, F \equiv J$, where

$$
F=J^{2} / G
$$

Also, since $\left\{y \in H_{0}^{1}(0, \infty): y=\beta y_{0}, \beta\right.$ a scalar $\}$ is a closed subspace of $H_{0}^{1}(0, \infty)$, Theorem 4.2 implies that for $m$ large enough there exists a nonzero scalar $\beta_{m}$ and a function $\epsilon_{m} \in H_{0}^{1}(0, \infty)$ such that

$$
w_{m}=\beta_{m}\left(y_{0}+\epsilon_{m}\right)
$$

and

$$
\left\langle y_{0}, \epsilon_{m}\right\rangle=0
$$

where $\langle\cdot, *\rangle$ is the inner product in $H_{0}^{1}$. 
Since $y_{0}$ and $\epsilon_{m}$ satisfy the properties of $y_{n}$ and $\epsilon$ of Theorem 2.4, we have

$$
6 \int_{0}^{\infty} y_{0}^{2} \epsilon_{m}^{2} r^{-2} d r<(2-\kappa)\left\|\epsilon_{m}\right\|_{1,2}^{2},
$$

where $\kappa$ is as in (2.5). The proof of the lemma which follows is deleted.

Lemma 4.3. Let $\left\{\epsilon_{m}\right\}$ be as in (4.15)-(4.16). Then

$$
\int_{0}^{\infty} y_{0} \epsilon_{m}^{3} r^{-2} d r, \int_{0}^{\infty} \epsilon_{m}^{4} r^{-2} d r=O\left(\left\|\epsilon_{m}\right\|_{1,2}^{3}\right) \text { as } m \rightarrow \infty
$$

THEOREM 4.4. There exists a positive constant $C$ independent of $m$ such that

$$
\left\|w_{m}-y_{0}\right\|_{1,2} \leqslant C m^{3 / 8} \exp \left(-.5 \beta m^{1 / 2}\right) \text { for all } m \geqslant 1 \text {, }
$$

where $\beta$ is as in Lemma 4.1 .

Proof. For $\epsilon_{m}$ as in (4.15),

$$
\begin{aligned}
G\left(y_{0}+\epsilon_{m}\right)= & \int_{0}^{\infty}\left(y_{0}+\epsilon_{m}\right)^{4} r^{-2} d r \\
= & \int_{0}^{\infty} y_{0}^{4} r^{-2} d r+4 \int_{0}^{\infty} y_{0}^{3} \epsilon_{m} r^{-2} d r+6 \int_{0}^{\infty} y_{0}^{2} \epsilon_{m}^{2} r^{-2} d r \\
& +4 \int_{0}^{\infty} y_{0} \epsilon_{m}^{3} r^{-2} d r+\int_{0}^{\infty} \epsilon_{m}^{4} r^{-2} d r .
\end{aligned}
$$

Hence, applying (4.17), and (4.18), we see that for large $m$,

$$
G\left(y_{0}+\epsilon_{m}\right)<J y_{0}+4\left\langle y_{0}, \epsilon_{m}\right\rangle+(2-\kappa)\left\|\epsilon_{m}\right\|_{1,2}+O\left(\left\|\epsilon_{m}\right\|_{1,2}^{3}\right) .
$$

So evaluation of $F$ gives us

$$
\begin{aligned}
F\left(y_{0}+\epsilon_{m}\right) & =\left[J\left(y_{0}+\epsilon_{m}\right)\right]^{2} / G\left(y_{0}+\epsilon_{m}\right) \\
& >\left[\left(J y_{0}\right)^{2}+2\left\|\epsilon_{m}\right\|_{1,2}^{2} J y_{0}+\left\|\epsilon_{m}\right\|_{1,2}^{4}\right]\left[J y_{0}+(2-\kappa)\left\|\epsilon_{m}\right\|_{1,2}^{2}\right. \\
& \left.+O\left(\left\|\epsilon_{m}\right\|_{1,2}^{3}\right)\right]^{-1} \\
& =J y_{0}+\kappa\left\|\epsilon_{m}\right\|_{1,2}^{2}+O\left(\left\|\epsilon_{m}\right\|_{1,2}^{3}\right),
\end{aligned}
$$

for $m$ large enough. Thus, we have for all large $m$,

$$
J w_{m}-J y_{0}=F\left(y_{0}+\epsilon_{m}\right)-J y_{0}>\kappa\left\|\epsilon_{m}\right\|_{1,2}^{2}+O\left(\left\|\epsilon_{m}\right\|_{1,2}^{3}\right) .
$$

From this, it follows

$$
\left\|\epsilon_{m}\right\|_{1,2}^{2}=O\left(J w_{m}-J y_{0}\right) \quad \text { as } m \rightarrow \infty .
$$

Now, we set $\delta_{m}=\beta_{m} \epsilon_{m}$ and note that $\beta_{m} \rightarrow 1$ as $m \rightarrow \infty$. Then

$$
\left\|\delta_{m}\right\|_{1,2}^{2}=O\left(J w_{m}-J y_{0}\right) \text { as } m \rightarrow \infty \text {. }
$$

Next, we have

$$
J w_{m}-J y_{0}=\left(\beta_{m}^{2}-1\right)\left\|y_{0}\right\|_{1,2}^{2}+\left\|\delta_{m}\right\|_{1,2}^{2},
$$

and so $\left|\beta_{m}-1\right|=O\left(J w_{m}-J y_{0}\right)$, as $m \rightarrow \infty$. Hence,

$$
\left(\beta_{m}-1\right)^{2}=O\left(J w_{m}-J y_{0}\right) \text { as } m \rightarrow \infty .
$$


Finally, since

$$
\left\|w_{m}-y_{0}\right\|_{1,2}^{2}=\left(\beta_{m}-1\right)^{2}\left\|y_{0}\right\|_{1,2}^{2}+\left\|\delta_{m}\right\|_{1,2}^{2},
$$

we obtain

$$
\left\|w_{m}-y_{0}\right\|_{1,2}^{2}=O\left(J w_{m}-J y_{0}\right) \text { as } m \rightarrow \infty .
$$

Applying Lemma 4.1, we obtain (4.19).

5. Numerical Results. Define $J_{m}$ and $G_{m}$ on $R_{n}$ by

$$
J_{m}\left(a_{1}, a_{2}, \ldots, a_{m}\right)=2 h_{m} \sum_{k=1}^{m} a_{k}^{2}+h_{m}^{-1} \sum_{k=1}^{m} \sum_{j=1}^{m} s_{k, j} a_{k} a_{j}
$$

and

$$
G_{m}\left(a_{1}, a_{2}, \ldots, a_{m}\right)=2 h_{m}^{-1} \sum_{k=1}^{\infty} k^{-2} w^{4}\left(k h_{m} / 2\right)
$$

where

$$
s_{k, j}= \begin{cases}2 \pi^{2} / 3-k^{-2} & \text { if } k=j \\ 4(-1)^{k+j}\left[(k-j)^{-2}-(k+j)^{-2}\right] & \text { if } k \neq j\end{cases}
$$

and

$$
w(r)=\sum_{k=1}^{m} a_{k}[S(k, h)(r)-S(-k, h)(r)] .
$$

Then Theorem 3.2 implies that

$$
w_{m}=\sum_{k=1}^{m} a_{k}^{*}[S(k, h)-S(-k, h)],
$$

where $\left(a_{1}^{*}, a_{2}^{*}, \ldots, a_{m}^{*}\right)$ is that element of

$$
A_{m} \equiv\left\{\left(a_{1}, a_{2}, \ldots, a_{m}\right): a_{k} \geqslant 0,1 \leqslant k \leqslant m ; a_{k} \neq 0 \text { for some } k ; J_{m}=G_{m}\right\}
$$

for which $J_{m}$ is minimized.

This minimization problem was solved using Newton's method with a Lagrange multiplier. The error bound of Theorem 4.4 depends on $\beta=\min (\pi d / \gamma, \alpha \gamma)$. In Section 3 it was noted that $\alpha=1$, and the proof of Theorem 3.1 (which was deleted) indicates that $d \geqslant \sqrt{6} /\left[1+\dot{y}_{0}(0)\right]$. In [2], $\dot{y}_{0}(0)$ is estimated to be 4.2 . Taking $\alpha=1, \dot{y}_{0}(0)=4$ and $\gamma=1.37$, we obtain $d=.59$ and $\beta=1.37$. These estimates yield the following values of $m^{3 / 8} \exp \left(-.5 \beta m^{1 / 2}\right)$ :

$\begin{array}{lc}\underline{m} & m^{3 / 8} \exp \left(-.69 m^{1 / 2}\right) \\ 50 & .0330 \\ 60 & .0222 \\ 70 & .0153\end{array}$


The approximation $w_{m}$ was obtained for each of the three cases listed above. The results for the case $m=70$ are given in Table 1. The accuracy was estimated by comparing successive approximations for $m=50$ and $m=60$. For $m=60, C$ was then estimated to be .00217 in the bound $C m^{3 / 8} \exp \left(-.69 \mathrm{~m}^{1 / 2}\right)$; this value of $C$ was used to obtain the "estimated accuracy" .000033 for $m=70$.

\section{TABLE 1}

Results for $m=70, h_{70}=1.37(70)^{-1 / 2}$

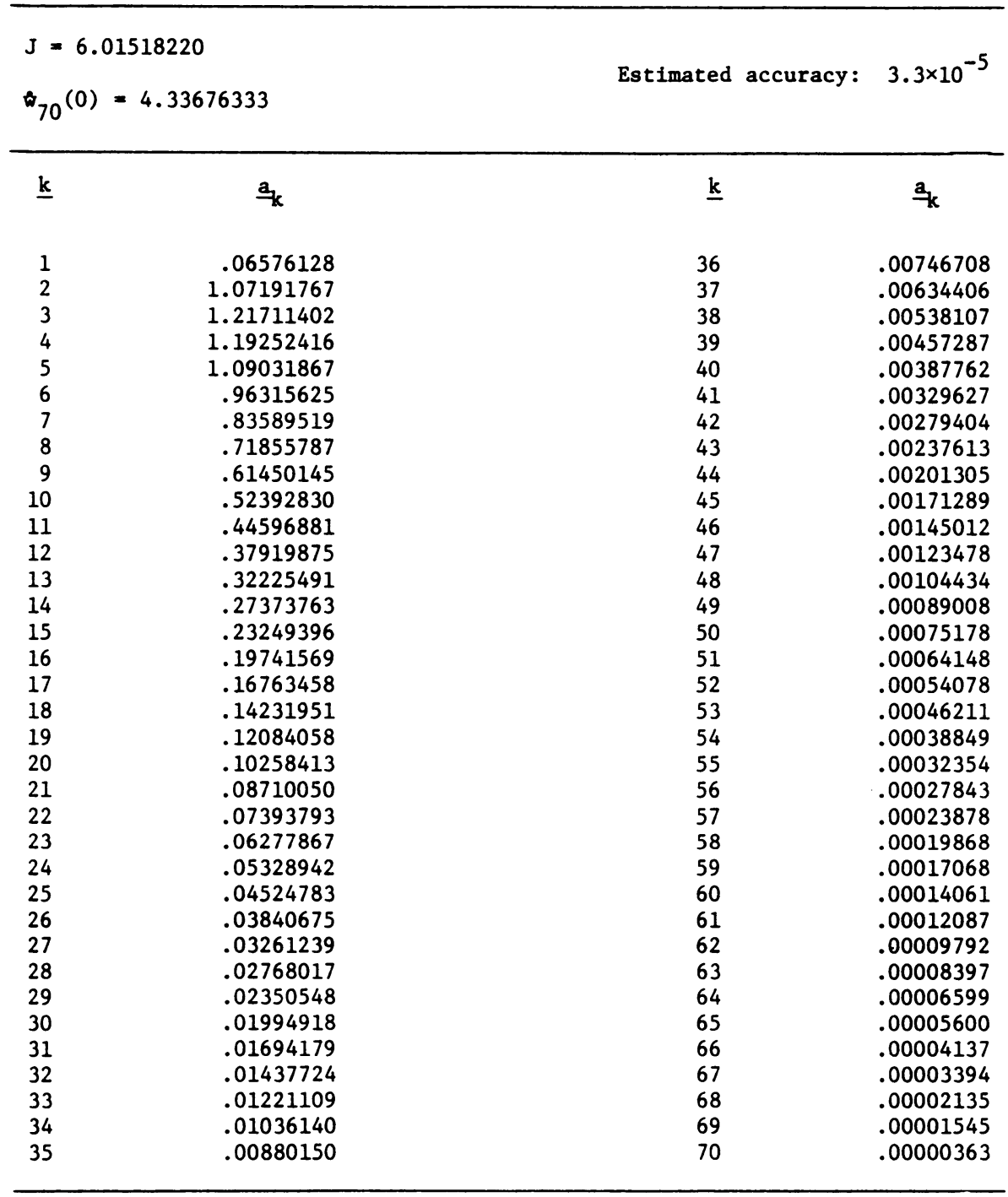

6. Concluding Remarks. We cannot close without reiterating that the order of convergence obtained in Section 4 is the best that can be obtained with known approximation techniques. If one were to use splines or other standard approximating functions, the rate of convergence would be $O\left(\mathrm{~m}^{-p}\right)$, for some integer $p$. Our rate of convergence is faster than $O\left(\mathrm{~m}^{-p}\right)$, for every positive $p$. For example, if 8-place accuracy were required, over 11,000 unknowns might be needed on the interval $[0,10]$ if $p$ were 3; at most 500 unknowns would be needed in our approach. 
The method presented here for $y_{0}$ could be perturbed slightly to obtain $y_{1}$. However, for $n \geqslant 1$ it would be more natural to use an approach based on the results given in [8]. That is, on each interval $\left(r_{j}, r_{j+1}\right)$ of Theorem 2.1, use the composition of a cardinal function and a conformal mapping to approximate $y_{n}$.

Finally, Eq. (1.4) is one of many equations of the form $\ddot{y}-y+y F\left(y^{2}, r\right)=0$. In [13], Ryder gives results for the more general problem. Our method may be employed once analyticity, uniqueness and exponential decay of solutions has been verified.

Department of Computer and Information Sciences State University College

Potsdam, New York 13676

1. C. V. COFFMAN, "Uniqueness of the ground state solution for $\Delta u-u+u^{3}=0$ and a variational characterization of other solutions," Arch. Rational Mech. Anal., v. 46, 1972, pp. $81-92$.

2. J. CHAUVETTE \& F. STENGER, "The approximate solution of the nonlinear equation $\Delta u=u-u^{3}, " J$. Math. Anal. Appl., v. 51. 1975, pp. 229-242.

3. L. COLLATZ, Functional Analysis and Numerical Mathematics, Academic Press, New York, 1966.

4. G. W. DAREWICH \& H. SCHIFF, "Particle solutions of a class of nonlinear field equations," J. Mathematical Phys., v. 8, 1967, pp. 1479-1482.

5. R. FINKELSTEIN, R. LE LEVIER \& M. RUDERMAN, "Nonlinear spinor fields," Phys. Rev., v. 83, 1950, pp. 326-332.

6. E. HILLE, Analytic Function Theory, Vol. 2, Blaisdell, Waltham, Mass., 1962.

7. IMSL Library 2, Edition 4 (Fortran V), International Mathematical and Statistical Libraries, Inc., Houston, Texas, 1974.

8. L. LUNDIN \& F. STENGER, "Cardinal type approximation of a function and its derivatives," SIAM J. Math. Anal., v. 10, 1979, pp. 139-160.

9. J. MCNAMEE, F. STENGER \& E. L. WHITNEY, "Whittaker's cardinal function in retrospect," Math. Comp., v. 25, 1963, pp. 141-154.

10. Z. NEHARI, "On a nonlinear differential equation arising in nuclear physics," Proc. Roy. Irish Acad. Sect. A, v. 62, 1963, pp. 117-135.

11. P. D. ROBINSON, "Extremum principles for the equation $\nabla \phi=\phi-\phi^{3}$, "J. Mathematical Phys., v. 12, 1971, pp. 23-28.

12. W. RUDIN, Real and Complex Analysis, McGraw-Hill, New York, 1966.

13. G. H. RYDER, "Boundary value problems for a class of nonlinear differential equations," Pacific J. Math., v. 22, 1967, pp. 477-503. 277-286.

14. H. SCHIFF, “A classical theory of bosons," Proc. Roy. Soc. Ser. A, v. 269, 1962, pp.

15. F. STENGER, Convergence and Error of the Bubnov-Galerkin Method, SIAM Conf. on Ordinary Differential Equations, Fall 1972.

16. F. STENGER, “Approximation via Whittaker's cardinal function," J. Approximation Theory, v. 17, 1976, pp. 222-240. mitted.)

17. F. STENGER, “Optimal convergence of minimum norm approximations in $H_{p}$." (Sub-

18. J. L. SYNGE, "On a certain nonlinear differential equation," Proc. Roy. Irish Acad. Sect. $A$, v. 62, 1961, pp. 17-41.

19. P. G. CIARLET, M. H. SCHULTZ \& R. S. VARGA, "Numerical methods of high-order accuracy for nonlinear problems, III. Eigenvalue problems," Numer. Math., v. 12, 1968, pp. 120133.

20. K. YOSIDA, Functional Analysis, Springer-Verlag, New York, 1966.

21. E. T. WHITTAKER, "On the functions which are represented by the expansions of the interpolation theory," Proc. Roy. Soc. Edinburgh Sect. A, v. 35, 1915 , pp. 181-194.

22. J. M. WHITTAKER, "On the cardinal function of interpolation theory," Proc. Edinburgh Math. Soc. Ser. I (2), 1927, pp. 41-46. 